\title{
Effects of serum vitamin D level on ventricular repolarization in children and adolescents
}

\author{
Bekdas $\mathrm{M}^{1}$, Inanir $\mathrm{M}^{2}$, Ilhan $\mathrm{Z}^{1}$, Ildes $\mathrm{E}^{1}$ \\ Department of Pediatrics, Bolu Abant Izzet Baysal University Faculty of Medicine, Bolu, Turkey. \\ merbek14@yahoo.com
}

\begin{abstract}
OBJECTIVES: Low levels of vitamin D are known to increase cardiovascular mortality due to different risks. We aimed to examine whether low vitamin $\mathrm{D}$ levels in children and adolescents cause ventricular repolarization changes.

METHODS: Sixtyseven healthy cases aged between 3.5 and 17 years were included. According to levels of vitamin $D$, cases were grouped as sufficient $(n=44)$, insufficient $(n=13)$ and deficiency $(n=10)$. Ventricular repolarization parameters were measured manually.

RESULTS: Levels of vitamin D were different for cases with insufficiency and deficiency compared to sufficiency ones ( $102 \pm 54.9$ vs $24.4 \pm 7 \mathrm{ng} / \mathrm{ml}, \mathrm{p}<0.001$ and $102 \pm 54.9$ vs $16.6 \pm 8.3 \mathrm{ng} / \mathrm{ml}$, respectively, $\mathrm{p}<0.001)$. In the insufficient group the following parameters were different from sufficient group: QTmean (357.8 \pm 25.3 vs $332 \pm 29.9 \mathrm{~ms}, p=0.012)$, JTc $(310.8 \pm 20.2$ vs $331.6 \pm 21 \mathrm{~ms}, p=0.005)$ and Tpe/QT $(0.2 \pm 0.02$ vs $0.22 \pm 0.02$, $p=0.02)$. It was found that the level of serum vitamin $D$ correlated with JTc $(r=-0.37, p=0.002)$, Tp-e $(r=-0.29$, $p=0.015)$ and Tp-e/QT $(r=-0.24, p=0.047)$. In the linear regression analysis, it was found that dropping level of vitamin $D$ below normal was an independent risk factor for prolonged duration of JTC $(p=0.015)$.

CONCLUSIONS: Decline in vitamin D levels causes ventricular repolarization anomaly. As the decrease in vitamin $\mathrm{D}$ levels deepens, repolarization times become longer. These results could indicate that decrease in vitamin D levels can increase sensitivity to ventricular arrhythmias (Tab. 3, Ref. 45). Text in PDF www.elis.sk KEY WORDS: adolescent, arrhythmia, child, vitamin D, ventricular repolarization.
\end{abstract}

\section{Introduction}

Vitamin D is synthesized as endogenous in the body (1), although its deficiency remains an important public health problem. Vitamin D is necessary for normal calcium and phosphorus metabolism, thanks to this hormone continuity of healthy bone mineralization is ensured. However, vitamin D receptor (VDR) was found in different cells such as cardiomyocytes (1). Thanks to these receptors, Vitamin D protects the cardiovascular system by regulating endothelial functions, suppressing atherogenic $\mathrm{T}$ lymphocytes and inhibiting vascular smooth muscle reproduction $(2,3)$. Studies have shown that low vitamin D levels are linked to cardiovascular risks such as diabetes mellitus, dyslipidemia, hypertension (4) and increased cardiovascular mortality (5).

Sudden cardiac death is the clinical picture that leads to death within an hour of the onset of acute cardiac symptoms and causes

${ }^{1}$ Department of Pediatrics, Bolu Abant Izzet Baysal University Faculty of Medicine, Bolu, Turkey, and ${ }^{2}$ Department of Cardiology, Bolu Abant Izzet Baysal University Faculty of Medicine, Bolu, Turkey

Address for correspondence: M. Bekdas, Assoc Prof, Department of Pediatrics, Bolu Abant Izzet Baysal University Faculty of Medicine, Bolu 14030, Turkey.

Phone/Fax:+903742535544

Acknowledgements: This study was supported by the Research Fund of the Abant Izzet Baysal University, Turkey (Project no: 2019.08.01.1419). the death of approximately 400,000 people each year in the United States. It is predicted that the incidence of this clinical picture that consists mostly of males is 60/100.000. This clinical picture is responsible for $5.6-15 \%$ of all deaths in western societies (6). Sudden cardiac death is rare in childhood and its frequency increases with age. Due to late notices, it is important to recognize those with noncardiac causes at the early stage. With this purpose it has been stated that changes in ventricular repolarization (7) happening at the distance between the beginning of the QRS complex and the end of the T wave (QT) in the ECG could be used to predict malignant arrhythmias (8). Tp-e interval, which is also the interval between $T$ wave peak and end point, has been used in recent years to show ventricular repolarization disorder (9). The JT interval, which is the distance between the end of the QRS wave (J point) and the end of the $\mathrm{T}$ wave is also a useful marker for defining the risk of arrhythmia (10).

One task of VDRs in cardiomyocytes is to regulate the operation and contra activation of voltage-dependent calcium channels along the cell membrane (1). In the literature, it has been shown that ventricular repolarization markers become impaired in adults with low vitamin D levels (11), but we have not been able to detect such a study done in childhood. In this study, our aim is to examine whether low serum vitamin D levels in children and adolescents cause ventricular repolarization changes, which are indicative of ventricular arrhythmia. 


\section{Patients and methods}

In this single-centric study, 67 healthy children and adolescents between the ages of 3.5 and 17 years who have visited our hospital were included. These cases consisted of those who came for routine follow-up and in none of these cases was a history of rickets, obesity, acute infection or chronic disease (congenital/acquired heart disease, chronic liver disease, chronic kidney failure, collagen tissue disease, chronic inflammatory disease), vitamin D use or history of the use of drugs causing arrhythmia. When the cases visited the out-patient department, written informed consent was taken from them and/or their parents.

A detailed medical history was taken from those who agreed to participate in the study and physical examinations were performed and anthropometric measurements were performed. Biochemical tests were performed. Serum was separated and stored at $-80^{\circ} \mathrm{C}$ to measure vitamin $\mathrm{D}$ levels, by using human Vitamin D (SinoGeneClon Biotech Co. Ltd) kit Elisa method was employed, cases were classified according to vitamin D level. Serum vitamin D level is considered to be sufficiency at $>20 \mathrm{ng} / \mathrm{ml}$, insufficiency at $12-20 \mathrm{ng} / \mathrm{ml}$ and deficiency is classified as $<12 \mathrm{ng} / \mathrm{ml}$ (12).

ECGs of all cases were recorded in our hospital during examination. For ECG shots, patients were rested for 10 minutes first, and then their shots were taken in the bed position, with a twelve-channel ECG device (Nihon Kohen Cardiofax ECG$1950 \mathrm{VET}$ ) at $10 \mathrm{~mm} / \mathrm{mV}$ amplitude and $25 \mathrm{~mm} / \mathrm{s}$. The ECG shutter time is $10 \mathrm{sec}-$ onds, during which 4-6 heartbeats were obtained per channel, depending on the pulse. From the ECGs taken, Tp-e, QT and JT, as well as QT and JT's pulse-corrected forms QTc and JTc were calculated, the calculations were done manually by the cardiologist with the help of a magnifying glass (TorQ $150 \mathrm{~mm}$ Digital Caliper LCD).

SPSS (Statistical Package for Social Sciences) version 21 program was used for statistical evaluation. Numerical data was expressed as average $\pm \mathrm{SD}$, and categorical data was expressed as numbers and percentages. One-Way Anova and Bonferroni tests were used in the analysis of the differences between the groups. $\mathrm{p}<0.05$ was used as a level of significance. Linear regression analysis was performed to determine ECG parameters where vitamin D level was effective, so variables with $\mathrm{p}<0.1$ were used in correlation analysis. moglobin)
Tab. 1. Demographic characteristics and laboratory findings of patients grouped by serum

\begin{tabular}{lcccccc}
\hline Features & $\begin{array}{c}\text { Sufficiency } \\
(\mathrm{n}=44)^{1}\end{array}$ & $\begin{array}{c}\text { Insufficiency } \\
(\mathrm{n}=13)^{2}\end{array}$ & $\begin{array}{c}\text { Deficiency } \\
(\mathrm{n}=10)^{3}\end{array}$ & $\mathrm{p}^{1-2}$ & $\mathrm{p}^{1-3}$ & $\mathrm{p}^{2-3}$ \\
\hline Age (years) & $11 \pm 2.9$ & $10.2 \pm 3.7$ & $12.2 \pm 4.1$ & 0.99 & 0.83 & 0.45 \\
Gender (M/F) (n) & $22 / 22$ & $5 / 8$ & $7 / 3$ & 0.86 & 0.32 & 0.46 \\
Age group (C/A)(n) & $26 / 18$ & $8 / 5$ & $3 / 7$ & 0.37 & 0.21 & 0.16 \\
Weight (kg) & $37.4 \pm 12.8$ & $42.8 \pm 12.8$ & $42.6 \pm 6.4$ & 0.99 & 0.98 & 0.92 \\
Height (cm) & $144.4 \pm 15.2$ & $152 \pm 10.6$ & $151.7 \pm 9.6$ & 0.7 & 0.99 & 0.97 \\
BMI & $17.4 \pm 3.3$ & $18.1 \pm 3.2$ & $18.5 \pm 2.7$ & 0.95 & 0.99 & 0.94 \\
Systolic BP(mmHg) & $101.3 \pm 13.4$ & $106.6 \pm 15$ & $102.5 \pm 9.5$ & 0.99 & 0.95 & 0.96 \\
DiastolicBP(mmHg) & $63.6 \pm 9.6$ & $66.6 \pm 10.3$ & $65 \pm 12.9$ & 0.93 & 0.94 & 0.99 \\
Hgb (g/dl) & $13.2 \pm 0.8$ & $13.4 \pm 0.8$ & $13.8 \pm 0.8$ & 0.99 & 0.19 & 0.76 \\
Sodium (mmol/l) & $137.9 \pm 6.2$ & $139.6 \pm 5.3$ & $140.4 \pm 2$ & 0.91 & 0.64 & 0.99 \\
Potassium (mmol/l) & $4.4 \pm 0.2$ & $4.5 \pm 0.5$ & $4.6 \pm 0.4$ & 0.58 & 0.2 & 0.98 \\
Calcium (mg/dl) & $9.8 \pm 0.3$ & $10 \pm 0.4$ & $9.9 \pm 0.5$ & 0.91 & 0.99 & 0.94 \\
Magnesium (mg/dl) & $2.1 \pm 0.1$ & $2 \pm 0.1$ & $1.9 \pm 0.2$ & 0.99 & 0.97 & 0.95 \\
Vitamin D (ng/ml) & $102 \pm 54.9$ & $24.4 \pm 7$ & $16.6 \pm 8.3$ & $<\mathbf{0 . 0 0 1}$ & $<\mathbf{0 . 0 0 1}$ & 0.98
\end{tabular}

(M - Male, F - Female, C - Child, A - Adolescent, BMI - Body mass index, BP - Blood pressure, Hgb - He-

Tab. 2. ECG findings of patients grouped by serum vitamin D level.

\begin{tabular}{lcccccc}
\hline Features & $\begin{array}{c}\text { Sufficiency } \\
(\mathrm{n}=44)^{1}\end{array}$ & $\begin{array}{c}\text { Insufficiency } \\
(\mathrm{n}=13)^{2}\end{array}$ & $\begin{array}{c}\text { Deficiency } \\
(\mathrm{n}=10)^{3}\end{array}$ & $\mathrm{p}^{1-2}$ & $\mathrm{p}^{1-3}$ & $\mathrm{p}^{2-3}$ \\
\hline QRS (ms) & $84.1 \pm 10.5$ & $77.6 \pm 9.6$ & $82.8 \pm 8.5$ & 0.14 & 0.99 & 0.7 \\
QTmax(ms) & $365.2 \pm 25.5$ & $339.8 \pm 30.8$ & $368.5 \pm 33.1$ & $\mathbf{0 . 0 1 5}$ & 0.99 & 0.051 \\
QTmin(ms) & $350.4 \pm 25.5$ & $324.2 \pm 29.6$ & $350 \pm 33.2$ & $\mathbf{0 . 0 1 1}$ & 0.99 & 0.088 \\
Pulse (/min) & $81.9 \pm 13.4$ & $101 \pm 18.7$ & $77.6 \pm 19.8$ & $\mathbf{0 . 0 0 1}$ & 0.98 & $\mathbf{0 . 0 0 2}$ \\
RR(second) & $0.75 \pm 0.12$ & $0.61 \pm 0.11$ & $0.81 \pm 0.17$ & $\mathbf{0 . 0 0 5}$ & 0.6 & $\mathbf{0 . 0 0 3}$ \\
QTav (ms) & $357.8 \pm 25.3$ & $332 \pm 29.9$ & $359.3 \pm 33$ & $\mathbf{0 . 0 1 2}$ & 0.98 & 0.06 \\
QTc (ms) & $414.8 \pm 18$ & $426.3 \pm 25.1$ & $402.6 \pm 21.1$ & 0.22 & 0.25 & $\mathbf{0 . 0 1 9}$ \\
QTd (ms) & $14.8 \pm 6.5$ & $15.6 \pm 7.6$ & $18.4 \pm 5.9$ & 0.98 & 0.37 & 0.96 \\
QTdc (ms) & $17.3 \pm 8.2$ & $19.8 \pm 9.1$ & $20.7 \pm 6.6$ & 0.98 & 0.74 & 0.98 \\
JT (ms) & $268.1 \pm 24.1$ & $258.4 \pm 25.8$ & $278.5 \pm 31.4$ & 0.7 & 0.75 & 0.2 \\
JTc (ms) & $310.8 \pm 20.2$ & $331.6 \pm 21$ & $311.7 \pm 19.3$ & $\mathbf{0 . 0 0 5}$ & 0.97 & 0.067 \\
Tp-e (ms) & $71.6 \pm 6.7$ & $74.4 \pm 9.2$ & $86.2 \pm 10.6$ & 0.82 & $<\mathbf{0 . 0 0 1}$ & $\mathbf{0 . 0 0 2}$ \\
Tp-e/QT & $0.2 \pm 0.02$ & $0.22 \pm 0.02$ & $0.24 \pm 0.03$ & $\mathbf{0 . 0 2}$ & $<\mathbf{0 . 0 0 1}$ & 0.36 \\
Tp-e/QTc & $0.17 \pm 0.01$ & $0.17 \pm 0.01$ & $0.21 \pm 0.02$ & 0.98 & $<\mathbf{0 . 0 0 1}$ & $<\mathbf{0 . 0 0 1}$ \\
Tp-e/JT & $0.26 \pm 0.03$ & $0.28 \pm 0.03$ & $0.31 \pm 0.05$ & 0.31 & $\mathbf{0 . 0 0 3}$ & 0.33 \\
Tp-e/JTc & $0.23 \pm 0.02$ & $0.22 \pm 0.02$ & $0.27 \pm 0.02$ & 0.98 & $<\mathbf{0 . 0 0 1}$ & $<\mathbf{0 . 0 0 1}$ \\
\hline
\end{tabular}

(ms - Mili second, min - Minute, QRS duration - Ventricular depolarization time, QTmax - The longest time for ventricular depolarization and repolarization, QTmin - The shortest time showing ventricular depolarization and repolarization, RR - Distance between two R waves, QTav - Half of the QTmax + QTmin, QTc - corrected QT, QTd - QTmax minus QTmin, QTdc - Corrected QT dispersion, JT - QRS end (J point) and T wave end distance, JTc - Corrected JT, Tp-e - T wave end time with the peak point of the T wave)

\section{Results}

The study included 67 healthy cases, 37 (55.2\%) children between the ages of 3.5 and 17 years, and 30 (44.8\%) adolescents. Of the cases, 34 were male (50.7 \%) and 33 were girls (49.2 \%). According to the obtained serum vitamin D results, the cases were divided into three groups as sufficiency, insufficiency and deficiency. The demographic characteristics of the three groups were similar for vital findings and laboratory findings (excluding serum vitamin D) ( $>0.05)$. Serum Vitamin D levels of those in the sufficiency group were significantly different compared to those with insufficiency and deficiency (102 $\pm 54.9^{\prime}$ vs $24.4 \pm 7 \mathrm{ng} / \mathrm{ml}$, $\mathrm{p}<0.001$ and $102 \pm 54.9$ vs $16.6 \pm 8.3 \mathrm{ng} / \mathrm{ml}, \mathrm{p}<0.001$, respec- 
Tab. 3. Correlation analysis results of serum vitamin D.

\begin{tabular}{lcc}
\hline Features & $\mathrm{r}$ & $\mathrm{p}$ \\
\hline QTd & -0.23 & 0.058 \\
QTdc & -0.23 & 0.055 \\
JT & -0.21 & 0.084 \\
JTc & -0.37 & $\mathbf{0 . 0 0 2}$ \\
Tp-e & -0.29 & $\mathbf{0 . 0 1 5}$ \\
Tp-e/QT & -0.24 & $\mathbf{0 . 0 4 7}$ \\
Tp-e/QTc & -0.23 & 0.055 \\
\hline
\end{tabular}

(QTd - QTmax and QTmin difference, QTdc - Corrected QT dispersion, JT - QRS end (J point) and T wave end distance, JTc - Corrected JT, Tp-e - T wave end time with the peak point of the $\mathrm{T}$ wave)

tively). The level of serum vitamin $\mathrm{D}$ in the deficiency group was also different compared to those with insufficiency level, but this difference was not statistically significant (16.6 \pm 8.3 vs $24.4 \pm 7$ ng/ml, p = 0.98) (Tab. 1).

According to sufficiency of vitamin $\mathrm{D}$ level, those with vitamin D insufficiency had QT max (365.2 \pm 25.5 vs $339.8 \pm 30.8 \mathrm{~ms}, \mathrm{p}$ $=0.015)$, QT min (350.4 \pm 25.5 vs $324.2 \pm 29.6 \mathrm{~ms}, \mathrm{p}=0.011)$, QT mean (357.8 \pm 25.3 vs $332 \pm 29.9 \mathrm{~ms}, \mathrm{p}=0.012)$, JTc (310.8 \pm 20.2 vs $331.6 \pm 21 \mathrm{~ms}, \mathrm{p}=0.005)$ and Tp-e/QT $(0.2 \pm 0.02$ vs 0.22 $\pm 0.02, p=0.02$ ) values that were significantly different (Tab. 2).

According to sufficiency of vitamin $\mathrm{D}$ level, those with a deficient vitamin D level had Tp-e (71.6 \pm 6.7 vs $86.2 \pm 10.6 \mathrm{~ms}, \mathrm{p}$ $<0.001)$, Tp-e/QT (0.2 \pm 0.02 vs $0.24 \pm 0.03, \mathrm{p}<0.001)$, Tp-e/ QTc $(0.17 \pm 0.01$ vs $0.21 \pm 0.02, \mathrm{p}<0.001), \mathrm{Tp}-\mathrm{e} / \mathrm{JT}(0.26 \pm 0.03$ vs $0.31 \pm 0.05, \mathrm{p}=0.003)$ and Tp-e/JTc $(0.23 \pm 0.02$ vs $0.27 \pm$ $0.02, \mathrm{p}<0.001$ ) values that were significantly different (Tab. 2).

Serum vitamin $\mathrm{D}$ level showed statistically significant correlation with JTc $(r=-0.37, \mathrm{p}=0.002)$, Tp-e $(\mathrm{r}=-0.29, \mathrm{p}=0.015)$ and Tp-e/QT ( $r=-0.24, p=0.047)$ (Tab. 3).

Linear regression analysis was performed to determine the ECG parameters affected by vitamin D levels, and for this, variables with $\mathrm{p}<0.1$ were used in correlation analysis. Linear regression analysis found that only JTc was an independent risk factor (r2 = 0.21, B = 0.83, 95\% CI $(-1.4-(-0.17)), \mathrm{p}=0.015)$.

\section{Discussion}

As well as regulating calcium metabolism in the body, Vitamin D is necessary for many systems to operate effectively, and it demonstrates these effects through existing VDRs on different cells. These receptors have been detected in many cells of the cardiovascular system, including vascular smooth muscles, endothelial cells and cardiomyocytes. These receptors have been detected in many cells of the cardiovascular system, including vascular smooth muscles, endothelial cells and cardiomyocytes. Vitamin $\mathrm{D}$, combined with VDR, regulates the transcription of genes that control cell proliferation, cell apoptosis, oxidative stress, membrane transport, matrix homeostasis and cell adhesion $(13,14)$, in this way vitamin $\mathrm{D}$ is considered to have an important role in the pathogenesis of cardiovascular diseases.

Ion channels in the cardiomyocyte membrane are responsible for the potential for action. In the ventricular repolarization phase after ventricular warehouses, calcium channels are closed and potassium channels are opened $(15,16)$. In this respect, changes in vitamin $\mathrm{D}$ levels, which regulate electrolytes in the body, can also affect the electrophysiology and contractility of the heart. As in a study conducted in adults (17), vitamin D deficiency in children can cause heart failure and ultimately death (18). Also in an adult study, it has been shown that there is a relationship between low vitamin D and sudden cardiac death (19). Malignant ventricular arrhythmias are the most common cause of such sudden cardiac deaths (19). The lack of vitamin D can cause ventricular arrhythmia and sudden cardiac death, associated with ion channels disorders and autonomic dysfunction $(20,21)$.

The repolarization phase is a complex electrical event. Repolarization disorder in ventricular myocardium is considered the underlying cause of arrhythmogenesis (22). The use of indices showing anomalies at this stage will allow early detection of ventricular arrhythmias and, as a result, reduce the risk of sudden cardiac death. For this purpose, the QT interval was used as a traditional marker, but the QT interval may not always accurately indicate the risk of arrhythmia, because this value may vary according to the pulse, so in order to make a more accurate assessment the use of QTc that is the corrected form of QT interval according to pulse is recommended. While there are studies showing that prolonged QTc is associated with malignant ventricular arrhythmias and sudden death $(23,24)$, there are also studies which approach this relationship with skepticism $(25,26)$. Therefore, it is recommended to use different QT variability assessment methods in the estimate of arrhythmia risk (27). In recent years, new parameters have been introduced for this purpose, one of which is the Tp-e interval (28). The distance between the peak point of the $\mathrm{T}$ wave and the end of the $\mathrm{T}$ wave. Studies have shown a positive relationship between elongation in the Tp e interval and ventricular arrhythmia (29). Maury et al (30) showed that the Tp-e interval was significantly higher when sudden cardiac death occurred. In order to predict the ventricular arrhythmias that may develop early, it has been stated that QTc(23) and Tp-e (31) derived from these parameters and in single form could be used (32, 33, 34). However, there are studies that indicate that Tp-e and Tp-e/QT values are not different from those in asymptomatic ones and with malignant arrhythmias (35).

The JT interval, which is the distance between the end of the QRS wave (J point) and the end of the T wave, has been more specifically accepted for ventricular repolarization in recent years (36, 37), but JT may vary depending on the pulse, for more accurate use of JT, JTc which is the pulse-corrected form of this parameter has been recommended (38). In individuals with prolonged QRS intervals, Jets is considered a more suitable marker in the measurement of ventricular repolarization compared to the QTC interval (39). Studies in children and adults have shown a close relationship between the development of ventricular arrhythmias with the prolonged JTc as an indicator of myocardial repolarization abnormality $(37,40)$. Apart from Tp-e and JTc, the increase in Tp-e/JTc values derived from these parameters can also be used for this purpose (37).

As well as those who suggest that there is no relationship between vitamin D and QT intervals (41), there are those who state 
that vitamin D deficiency prolongs QT intervals and thus can lead to arrhythmias (42). In patients with low vitamin D, which is accompanied by symptoms such as type 2 diabetes, the QT interval was found to be prolonged as a marker of ventricular repolarization abnormality (43). In individuals taking antiepileptic drugs that cause vitamin D deficiency, the QT interval has been shown to grow (42). On the other hand, in healthy individuals, vitamin $\mathrm{D}$ levels have been shown to increase in those with no vitamin $\mathrm{D}$ deficiency or insufficient values such as Tp e, Tp e/QTc and Tp-e/JT(11).

There is only one study of new repolarization anomalies as an indicator of malignant ventricular arrhythmias with serum vitamin D level(11), no such study involving children in the literature has been detected. In our study, the values of JTc and Tp-e/QT, which are ventricular repolarization markers of those with insufficient vitamin D levels compared to of those with sufficient vitamin D levels, are increasing. When serum vitamin $\mathrm{D}$ levels decrease from insufficiency to minus, Tp-e, Tp-e/QTc and Tp-e/JTc values increase. These findings suggest that as the decrease in vitamin $\mathrm{D}$ levels deepens, it can further prolong the repolarization interval, thus increasing sensitivity to malignant ventricular arrhythmias.

We identified a negative correlation between serum vitamin D level and ventricular repolarization parameters, which showed that as serum vitamin D levels decreased, ventricular repolarization parameters such as JTc, Tp-e and Tp-E/QT were prolonged.

Prolonged ventricular repolarization is a cardiac calcium imbalance that indirectly impairs the function of the cyclic nucleotidegated channels and voltage-gated potassium $(44,45)$. Vitamin D is thought to have an effect on the JT interval by adjusting cardiac calcium balance and the function of potassium channels (42).

The limitations of our study are that it is single-centric study carried out with low number of individuals and that ECG findings for those with decreased Vitamin D levels have not been examined after treatment. For more precise results, multi-centric and wider series are needed.

As a result, the decrease in serum vitamin D levels causes ventricular repolarization anomaly in children and adolescents. As the decrease in vitamin D levels deepens, repolarization times become longer, especially JTc can be an important parameter that can show this disorder. These results suggest that the decrease in vitamin $\mathrm{D}$ levels can increase sensitivity to ventricular arrhythmias.

\section{References}

1. Zhao G, Simpson RU. Membrane localization, Caveolin-3 association and rapid actions of vitamin D receptor in cardiac myocytes. Steroids 2010. p. 555-559.

2. Dong J, Wong SL, Lau CW et al. Calcitriol protects renovascular function in hypertension by down-regulating angiotensin II type 1 receptors and reducing oxidative stress. Eur Heart J 2012; 33 (23): 2980-2990.

3. Al Mheid I, Patel RS, Tangpricha V, Quyyumi AA. Vitamin D and cardiovascular disease: is the evidence solid? Eur Heart J 2013; 34 (48): 3691-3698.

4. Pilz S, Tomaschitz A, März W et al. Vitamin D, cardiovascular disease and mortality. Clin Endocrinol 2011; 75: 575-584.
5. Grandi NC, Breitling LP, Brenner H. Vitamin D and cardiovascular disease: systematic review and meta-analysis of prospective studies. Prevent Med 51 (3-4): 228-233.

6. Stecker EC, Reinier K, Marijon E et al. Public health burden of sudden cardiac death in the United States. Circulat Arrhyth Electrophysiol 2014; 7 (2): 212-217.

7. Franz MR, Bargheer K, Rafflenbeul W, Haverich A, Lichtlen PR. Monophasic action potential mapping in human subjects with normal electrocardiograms: Direct evidence for the genesis of the T wave. Circulation 1987; 75 (2): 379-386.

8. Antzelevitch COA. Amplification of spatial dispersion of repolarization underlies sudden cardiac death associated with catecholaminergic polymorphic VT, long QT, short QT and Brugada syndromes. J Intern Med 2006; 259 (1): 48-58.

9. Kors JA, Ritsema van Eck HJ, van Herpen G. The meaning of the TpTe interval and its diagnostic value. J Electrocardiol 2008; 41 (6): 575-580.

10. Helbing WA, Roest AAW, Niezen RA et al. ECG predictors of ventricular arrhythmias and biventricular size and wall mass in tetralogy of Fallot with pulmonary regurgitation. Heart 2002; 88 (5): 515-520.

11. Bagrul D, Atik F. Association of vitamin D deficiency with ventricular repolarization abnormalities. Kardiol Pol 2019; 77 (9): 853-858.

12. Munns CF, Shaw N, Kiely M et al. Global Consensus Recommendations on Prevention and Management of Nutritional Rickets. Horm Res Paediatr 2016; 85 (2): 83-106.

13. Nibbelink KA, Tishkoff DX, Hershey SD, Rahman A, Simpson RU. 1,25 (OH)2-vitamin D3 actions on cell proliferation, size, gene expression, and receptor localization, in the HL-1 cardiac myocyte. J Steroid Biochem Mol Biol 2007; 103 (3-5): 533-537.

14. Tishkoff DX, Nibbelink KA, Holmberg KH, Dandu L, Simpson RU. Functional vitamin D receptor (VDR) in the T-tubules of cardiac myocytes: VDR knockout cardiomyocyte contractility. Endocrinology 2008; 149 (2): 558-564.

15. Behere SP, Weindling SN. Inherited arrhythmias: The cardiac channelopathies. Ann Pediatr Cardiol Medknow Publications 2015; 8: 210-220.

16. Kline J, Costantini O. Inherited Cardiac Arrhythmias and Channelopathies. Med Clin North Amer. 2019; 103 (5): 809-820.

17. D'Amore C, Marsico F, Parente A et al. Vitamin D Deficiency and Clinical Outcome in Patients With Chronic Heart Failure: A Review. Nutr Metab Cardiovasc Dis 2017; 27 (10): 837-849.

18. Maiya $\mathbf{S}$, Sullivan $\mathbf{I}$, Allgrove $\mathbf{J}$ et al. Hypocalcaemia and vitamin $D$ deficiency: An important but preventable, cause of life-threatening infant heart failure. Heart 2008; 94 (5): 581-584.

19. Deo R, Katz R, Shlipak MG et al. Vitamin D, parathyroid hormone, and sudden cardiac death: Results from the cardiovascular health study. Hypertension 2011; 58 (6): 1021-1018.

20. Bolland MJ, Grey A, Avenell A, Gamble GD, Reid IR. Calcium supplements with or without vitamin D and risk of cardiovascular events: Reanalysis of the Women's Health Initiative limited access dataset and meta-analysis. BMJ 2011; 342 (7804).

21. Mann MC, Hollenberg MD, Hanley DA, Ahmed SB. Vitamin D, the autonomic nervous system, and cardiovascular risk. Physiol Report Amer Physiol Soc 2015; 8.

22. Yan GX, Antzelevitch C. Cellular basis for the Brugada syndrome and other mechanisms of arrhythmogenesis associated with ST-segment elevation. Circulation 1999; 100 (15): 1660-1666. 
816-820

23. Locati ET, Bagliani G, Cecchi F, Johny H, Lunati M PC. Arrhythmias due to Inherited and Acquired Abnormalities of Ventricular Repolarization. Cardiac Electrophysiol Clin 2019; 11 (2): 345-362.

24. Galluzzo A, Gallo C, Battaglia A et al. Prolonged QT interval in ST-elevation myocardial infarction: predictors and prognostic value in medium-term follow-up. J Cardiovasc Med 2016; 17 (6): 440-445.

25. Roden DM. Drug-Induced Prolongation of the QT Interval. Wood AJJ, editor. New Engl J Med 2004 ; 350 (10): 1013-1022.

26. Grimm W, Christ M, Bach J, Müller H-H, Maisch B. Noninvasive arrhythmia risk stratification in idiopathic dilated cardiomyopathy: results of the Marburg Cardiomyopathy Study. Circulation 2003; 108 (23): 2883-2891.

27. Zareba W, Bayes de Luna A. QT dynamics and variability. Annals of noninvasive electrocardiology: the official journal of the International Society for Holter and Noninvasive Electrocardiology, Inc. 2005; 10 (2): 256-262.

28. Antzelevitch C, Sicouri S, Di Diego JM et al. Does Tpeak-Tend provide an index of transmural dispersion of repolarization? Heart Rhythm 2007; 4: 1114-1116.

29. Smetana P, Schmidt A, Zabel M et al. Assessment of repolarization heterogeneity for prediction of mortality in cardiovascular disease: Peak to the end of the $\mathrm{T}$ wave interval and nondipolar repolarization components. J Electrocardiol 2011; 44 (3): 301-308.

30. Maury P, Sacher F, Gourraud J-B et al. Increased Tpeak-Tend interval is highly and independently related to arrhythmic events in Brugada syndrome. Heart Rhythm 2015; 12 (12): 2469-2476.

31. Tse G, Gong M, Li CKH et al. T peak -T end, T peak -T end /QT ratio and $\mathrm{T}$ peak -T end dispersion for risk stratification in Brugada syndrome: A systematic review and meta-analysis. J Arrhythm 2018; 34 (6): 587-597.

32. Wang X, Zhang L, Gao C, Wu S, Zhu J. ST-segment elevation and the Tpeak-Tend/QT ratio predict the occurrence of malignant arrhythmia events in patients with vasospastic angina. J Electrocardiol 53: 52-56.

33. Alvarado-Serrano C, Ramos-Castro J, Pallàs-Areny R. Novel indices of ventricular repolarization to screen post myocardial infarction patients. Comput Biol Med 2006; 36 (5): 507-515.

34. Karaman K, Altunkaş F, Çetin M et al. New Markers for Ventricular Repolarization in Coronary Slow Flow: Tp-e Interval, Tp-e/QT Ratio, and Tp-e/QTc Ratio. Anna Noninvas Electrocardiol 2015; 20 (4): 338-344.
35. Mugnai G, Hunuk B, Hernandez-Ojeda J et al. Role of Electrocardiographic Tpeak-Tend for the Prediction of Ventricular Arrhythmic Events in the Brugada Syndrome. Amer J Cardiol 2017; 120 (8): 1332-1337.

36. Tse G, Yan BP. Traditional and novel electrocardiographic conduction and repolarization markers of sudden cardiac death. Europace. Oxford University Press 2017; 19: 712-721.

37. Inanir M, Sincer I, Erdal E, Gunes Y, Cosgun M, Mansiroglu AK. Evaluation of electrocardiographic ventricular repolarization parameters in extreme obesity. J Electrocardiol 2019; 53: 36-39.

38. El-Eraky H, Thomas SHL. Effects of sex on the pharmacokinetic and pharmacodynamic properties of quinidine. Brit J Clin Pharmacol 2003; 56 (2): 198-204.

39. Crow RS, Hannan PJ, Folsom AR. Prognostic Significance of Corrected QT and Corrected JT Interval for Incident Coronary Heart Disease in a General Population Sample Stratified by Presence or Absence of Wide QRS Complex: The ARIC Study With 13 Years of Follow-Up. Circulation 2003; 108 (16): 1985-1989.

40. Nigro G, Russo V, Di Salvo G et al. Increased heterogenity of ventricular repolarization in obese nonhypertensive children. PACE 2010; 33 (12): 1533-1539.

41. Zhang Y, Post WS, Dalal D et al. Serum 25-hydroxyvitamin D, calcium, phosphorus, and electrocardiographic QT interval duration: findings from NHANES III and ARIC. J Clin Endocrinol Metab 2011; 96 (6): 1873-1882.

42. Al-Nimer MS. The benefit of prescribing vitamin $D$ as add on therapy on the electrocardiographic changes in epileptic patients. Curr Clin Pharmacol 2018; 13 (1): 40-44.

43. Yetkin DO, Kucukkaya B, Turhan M, Oren M. The effect of 25-hydroxyvitamin D levels on QT interval duration and dispersion in type 2 diabetic patients. Croat Med J 2015; 56 (6): 525-530.

44. Powell KL, Jones NC, Kennard JT et al. HCN channelopathy and cardiac electrophysiologic dysfunction in genetic and acquired rat epilepsy models. Epilepsia 2014; 55: 609-620.

45. Bealer SL, Little JG, Metcalf CS, Brewster ALAA. Autonomic and cellular mechanisms mediating detrimental cardiac effects of status epilepticus. Epilepsy Res 2010; 91 (1): 66-73.

Received September 23, 2020. Accepted February 21, 2021. 